\title{
Modelling the Expansion of NGC 7027
}

\author{
J. R. Walsh ${ }^{1}$, G. Dudziak ${ }^{1}$ and N. A. Walton ${ }^{2}$ \\ ${ }^{1}$ European Southern Observatory; ${ }^{2}$ Royal Greenwich Observatory, La Palma
}

The high angular resolution and dynamic range of VLA radio continuum mapping of planetary nebulae has raised expansion parallax measurement to the most sensitive method for determining PN distances (Hajian et al 1995). In order to derive the distance, the proper motion in the plane of the sky must be compared to the expansion velocity measured from emission line profiles. For a spherical PN with a measured line of sight expansion velocity, the comparison is simple. However most PN are not spherical, and their axisymmetric structures may be tilted to the line of sight; the line of sight expansion velocity cannot therefore be used for distance estimation from expansion proper motion. 3-D kinematic modelling is required in order to determine the expansion velocity in the plane of the sky.

Imaging Fabry-Pérot observations of NGC 7027 were made with TAURUS-I and TAURUS-II on the $2.5 \mathrm{~m}$ INT and the $4.2 \mathrm{~m}$ WHT. Velocity cubes in He II 4686, [O III] $5007 \AA$, [O I] $6300 \AA, \mathrm{H} \alpha,[\mathrm{N} \mathrm{II}] 6583 \AA$ were obtained. The modelling method is similar to that applied to the TAURUS data of NGC 7139 (Walton, Walsh \& Sahu, 1990). A 3-D geometric form is assumed for the nebula and the variation of emission $v s$. radius is taken from the photoionization model of Middlemas (1991). A velocity law (viz. expansion velocity $v s$. nebular radius) can be applied to the 3-D emission model to produce a simulated TAURUS cube. A match was found between the observed TAURUS velocity cubes and a single velocity law (c.f. Roelfsema et al 1991) which provides fair fits to the $\mathrm{H} \alpha$, [N II] and [O III] data:

$V=-10+1400 \times r(p c) / D(k p c)$

where $\mathrm{V}$ is the recession velocity of matter from the central star, $\mathrm{r}$ the distance from the star and D the distance to the nebula.

This velocity law was applied to a prolate ellipsoid model tilted by $30^{\circ}$ to the plane of the sky (Masson, 1989, Model 3). Viewing this 3-D model at right angles to the line of sight, allows the (unobservable) expansion velocity in the plane of the sky to be directly determined. The line profile formed by viewing the minor axis expansion in the plane of the sky has a measured line splitting was $37.8 \mathrm{kms}^{-1}$, implying an expansion velocity of $18.9 \mathrm{kms}^{-1}$. Hajian et al (1993) derive a minor axis proper motion expansion of $5.25 \pm$

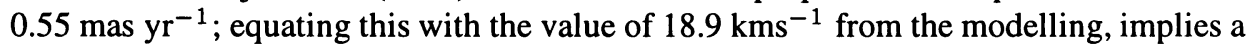
distance of 760 pc.

\section{REFERENCES}

Hajian, A. R., Terzian, Y., Bignell, C., 1993, AJ, 106, 1965

Hajian, A. R., Terzian, Y., Bignell, C., 1995, AJ, 109, 2600

Masson, C. R., 1989, ApJ, 336, 294

Middlemass, D., 1990, MNRAS, 244, 294

Roelfsema, P. R., Goss, W. M., Pottasch, S. R., Zijlstra, A. A., 1991, A\&A, 251, 611

Walton, N. A., Walsh, J. R., Sahu, K. C., 1990, A\&A, 230, 445 